\title{
A DERIVATIVE TEST FOR FINITE SOLUTIONS OF GAMES
}

\section{GLICKSBERG}

For a continuous real-valued function $M$ (called the payoff ${ }^{1}$ ) on the unit square, the minimax theorem of game theory states that there is a unique constant $v$ (the value) and probability distributions $f, g$ over $[0,1]$ (called maximizing and minimizing optimal strategies, respectively) for which

$$
\int_{0}^{1} M(x, y) d f(x) \geqq v \geqq \int_{0}^{1} M(x, y) d g(y) .
$$

At present there is no general method for finding these optimal strategies (which need not be unique), and, consequently, results which reduce the set of distributions among which one need search are desirable. Recently Samuel Karlin [3] has introduced a class of payoff functions $M$, related to convex payoff functions [1], for which optimal strategies with at most a finite number of points of increase always exist. The purpose of this note is to give a proof for all $n$ of the theorem concerning these functions which Karlin proved for $n \leqq 4$ :

THEOREM. If $\partial^{n} M / \partial y^{n}$ is continuous and is of one sign, then there is a maximizing optimal strategy with at most $n$ points of increase and a minimizing optimal strategy with at most $n / 2$ points of increase (counting, in this case, 0 and 1 as half points).

Proof. As is shown in [3], we need only consider the case in which the partial never vanishes, and clearly we may take $v=0$. Let $f_{0}$ be a fixed maximizing optimal strategy. From (1) it is apparent that any optimal $g$ places weight at only the minima of $k(y)=\int_{0}^{1} M(x, y) d f_{0}(x)$; since $k^{(n)}(y)=\int_{0}^{1}\left(\partial^{n} M / \partial y^{n}\right) d f_{0}(x) \neq 0$, a simple argument based on Rolle's Theorem shows there are at most $n / 2$ minimum points, counting 0 and 1 as half points, and the second assertion of the theorem follows. Furthermore, since $k^{(n)}(y) \neq 0$, it follows from Rolle's Theorem that the total number of roots of $k$, counting multiplicities, is at most $n$.

To establish the first assertion of the theorem we shall construct an auxiliary polynomial-like payoff function [2], the construction being based on this last observation. We first assert that there is a

Received by the editors April 9, 1953.

${ }^{1}$ For game-theoretic notation and motivation the reader is referred to [4, preface] and [5]. 
polynomial $p_{0}$ of degree less than $n$ for which, on $[0,1], k \geqq p_{0} \geqq 0$ and $k-p_{0}$ has exactly $n$ roots counting multiplicities. For consider the nonvoid set of polynomials $p$ of degree less than $n$ for which $k \geqq p \geqq 0$. Counting multiplicities, the number of roots of $k-p$ is at most $n$ (since $k^{(n)}-p^{(n)}=k^{(n)} \neq 0$ ) for these polynomials, so let $p_{0}$ be one for which the number of roots is maximum. If $k-p_{0}$ has roots $y_{i}$ of multiplicity $m_{i}, i=1, \cdots, r$, and $\sum m_{i}<n$, then, since the roots $y_{i}$ interior to $[0,1]$ are of even multiplicity, there is a polynomial $p_{1} \geqq 0$ (on $[0,1]$ ) of degree less than $n$ having just these roots and multiplicities. Consequently we may define the continuous function $\left(k-p_{0}\right) / p_{1}$ by setting

$$
\left(\left(k-p_{0}\right) / p_{1}\right)\left(y_{i}\right)=\left(k_{0}^{\left(m_{i}\right)}\left(y_{i}\right)-p_{0}^{\left(m_{i}\right)}\left(y_{i}\right)\right) / p_{1}^{\left(m_{i}\right)}\left(y_{i}\right)
$$

and clearly $\lambda=\min _{y}\left(\left(k-p_{0}\right) / p_{1}\right)(y)>0$. But then $k \geqq p_{0}+\lambda p_{1}$ $\geqq 0$, and, since $k-p_{0}-\lambda p_{1}$ can thus have at most $\sum m_{i}$ roots, counting multiplicities, we must have $k^{\left(m_{i}\right)}\left(y_{i}\right)-p_{0}^{\left(m_{i}\right)}\left(y_{i}\right)-\lambda p_{1}^{\left(m_{i}\right)}\left(y_{i}\right)$ $>0$ and $k(y)-p_{0}(y)-\lambda p_{1}(y)>0$ for $y \neq y_{i}, i=1, \cdots, r$, so that $\left(\left(k-p_{0}\right) / p_{1}\right)(y)>\lambda=\min \left(k-p_{0}\right) / p_{1}$, a contradiction. Thus we must have $\sum m_{i}=n$, so that $p_{0}$ is the desired polynomial.

Now it is easily seen that for each fixed $x$ one may find coefficients $a_{j}(x), j=0, \cdots, n-1$, for which $\phi_{x}(y)=M(x, y)-\sum_{j=0}^{n-1} a_{j}(x) y^{j}$ has the roots $y_{i}$ of multiplicity $m_{i}$. Moreover, this polynomial in $y$ is unique, ${ }^{2}$ so that, since we may write $n$ linear equations for the unknowns $a_{0}(x), \cdots, a_{n-1}(x)$, the matrix of coefficients in these equations is nonsingular, and the $a_{j}$ are seen to be continuous functions.

For any $x, \phi_{x}$ has a nonvanishing $n$th derivative, so that its only roots are the $y_{i}$ (of multiplicity $m_{i}$ ). Since those interior to $[0,1]$ are of even multiplicity, $\phi_{x}$ is of one sign and not identically zero-thus by continuity either

$$
M(x, y)-\sum_{j=0}^{n-1} a_{j}(x) y^{j} \geqq 0 \quad \text { for all } x, y
$$

or

$$
M(x, y)-\sum_{j=0}^{n-1} a_{j}(x) y^{j} \leqq 0 \quad \text { for all } x, y .
$$

But integration of this expression with respect to $f_{0}$ yields $k(y)$ $-\sum_{j=0}^{n-1}\left(\int_{0}^{1} a_{j}(x) d f_{0}(x)\right) y^{j}$, and since this function of $y$ has roots $y_{i}$

2 The difference of two such polynomials, of degree less than $n$, has $n$ roots, hence $=0$. 
of multiplicity $m_{i}$ (since this was true for each $\phi_{x}$ ), we must have $p_{0}(y)-\sum_{j=0}^{n-1}\left(\int_{0}^{1} a_{j}(x) d f_{0}(x)\right) y^{j}=0$, as a polynomial of degree less than $n$ with $n$ roots. Since $k(y)>p_{0}(y)$ for some $y,(2)$ must hold.

Now if $g_{0}$ is any minimizing optimal strategy, we have

$$
\begin{aligned}
\int_{0}^{1} \sum a_{j}(x) y^{j} d g_{0}(y) & \leqq \int_{0}^{1} M(x, y) d g_{0}(y) \leqq 0 \leqq p_{0}(y) \\
& =\int_{0}^{1} \sum a_{j}(x) y^{j} d f_{0}(x)
\end{aligned}
$$

so that the payoff function $\sum_{j=0}^{n-1} a_{j}(x) y^{j}$ has value $v=0$ (cf. (1)). In view of its polynomial-like character [2] it has a maximizing optimal strategy $f$ with at most $n$ points of increase, so that

$$
\int_{0}^{1} M(x, y) d f(x) \geqq \int_{0}^{1} \sum a_{j}(x) y^{i} d f(x) \geqq 0,
$$

and $f$ is a maximizing optimal strategy for $M$, which completes the proof.

A second theorem of Karlin now appears as a

COROLLARY. If $\partial^{n} M / \partial y^{n}$ is of one sign and never zero, and there is a $k$-dimensional set of minimizing optimal strategies, then there is a maximizing optimal strategy with at most $n-k$ points of increase.

For since $M(x, y) \geqq \sum a_{j}(x) y^{j}$ and both functions are payoffs with value $v=0$, if $g$ is a minimizing optimal strategy for $M$, then 0 $\geqq \int_{0}^{1} \sum a_{j}(x) y^{j} d g(y)$, i.e., $g$ is a minimizing optimal strategy for $\sum a_{j}(x) y^{i}$-thus this polynomial-like function has a $k$-dimensional set of minimizing optimal strategies, and, in view of the results of [2], has a maximizing optimal strategy with at most $n-k$ points of increase which, as before, is optimal for $M$.

\section{REFERENCES}

1. H. F. Bohnenblust, S. Karlin, and L. S. Shapley, Games with continuous convex payoff, "Contributions to the theory of games," Annals of Mathematics Studies, no. 24, pp. 181-192.

2. M. Dresher, S. Karlin, and L. S. Shapley, Polynomial games, "Contributions to the theory of games," Annals of Mathematics Studies, no. 24, pp. 161-180.

3. S. Karlin, On a class of games, "Contributions to the theory of games," vol. II, Annals of Mathematics Studies, no. 28, pp. 159-171.

4. H. W. Kuhn and A. W. Tucker, Contributions to the theory of games, Annals of Mathematics Studies, no. 24.

5. J. von Neumann and 0 . Morgenstern, Theory of games and economic behavior, Princeton, 1947.

RAND CORPoration 